\title{
A STOCHASTIC THEORY OF GRAIN GROWTH
}

\author{
I-WEI CHEN \\ Department of Materials Science and Engineering, The University of Michigan, \\ Ann Arbor, MI 48109, U.S.A.
}

(Received 30 January 1986; in revised form 8 October 1986)

\begin{abstract}
A stochastic theory of normal grain growth is proposed. The model is based on the concept that the migration of kinks and ledges should cause a Brownian motion of the grain boundary. This motion results in a drift of the grain size distribution to larger sizes. The kinetics of grain growth is thus related to the kinetics of kinks and ledges; specifically, via the rates of nucleation, recombination and sink annihilation. A variety of growth exponents are obtained from a scaling analysis, but only one universal grain size distribution is applicable in all cases. The specific predictions of this model are in total agreement with the recent computer simulations of domain growth, and are consistent with experimental observations of normal grain growth.
\end{abstract}

\begin{abstract}
Résumé--Nous proposons une théorie stochastique de la croissance normale du grain. Le modèle est basé sur l'idéc que la migration des crans et des marches provoque un mouvement brownien du joint de grains, ce qui aurait pour conséquence un déplacement de la répartition des tailles de grains vers des tailles plus grandes. La cinétique de croissance du grain est ainsi reliée à la cinétique des crans et des marches et plus précisément, à travers leurs vitesses de germination, à la cinétique de recombinaison et d'annihilation par puits. Nous obtenons divers exposants de croissance à partir d'une analyse d'échelle, mais une seule répartition universelle de tailles de grains est applicable à tous les cas. Les prévisions précises de ce modèle sont en complet accord avec les dernières simulations sur ordinateur de la croissance de domaines, et concordent avec les observations expérimentales de la croissance normale du grain.
\end{abstract}

\begin{abstract}
Zusammenfassung - Es wird eine stochastische Theorie des normalen Kornwachstums vorgeschlagen. Das Modell basiert aut dem Konzept, daB die Bewegung von Kinken und Stufen eine Brownsche Bewegung der Korngrenze verursacht. Diese Bewegung führt zu einer Drift der Verteilung der Korngrößen zu höheren Werten. Die Kinetik des Kornwachstums hängt dann mit der Kinetik der Kinken und Stufen zusammen, insbesondere über deren Bildungs-, Rekombinations- und Annihilationsraten. Aus ciner Skalierungsanalyse folgt eine Vielfalt von Wachstumsexponenten, jedoch ist in allen Fällen nur eine universelle Verteilung der Konrgrößen anwendbar. Die spezifischen Aussagen dieses Modells stimmen vollständig mit den jüngsten Computersimulationen des Domänenwachstums überein und sie sind verträglich mit den experimentellen Beobachtungen zum normalen Kornwachstum.
\end{abstract}

\section{INTRODUCTION}

There are two major theories of normal grain growth in the literature. The first, put forth by Hillert [1], is a thermodynamic approach which emphasizes the reduction of the boundary curvature as the main driving force for grain growth. The second, taken by Louat [2], is a kinetic approach which postulates the fluctuation of the grain size as the cause of the drift to larger sizes. In the case of constant mobilities, both theories predict a dependence of the grain size $R$ on the time $t$, in a form of $R \sim t^{\frac{1}{2}}$. Since few experiments on grain growth have yet reported such time dependence, it is commonly assumed that mobilities are grain-size dependent, presumably due to solute segregation, particle pinning, or changes of grainboundary structures. From such assumptions, it is possible to obtain $R \sim t^{n}$, with $n$ smaller than $\frac{1}{2}$ [3-6].

Until now, the above two approaches have been thought to be distinct from each other, and constructed on different physical bases. We now demonstrate that this is not so, using a simple example of a sinusoidal grain boundary. On this new realization, we proceed to develop a theory of grain growth using a unified approach. The theory gives predictions on the growth law which are in entire agreement with the recent computer simulations [7-11]. These predictions also appear to be consistent with experimental observations.

Consider first a bicrystal with a grain boundary which is curved in a sinusoidal manner, as illustrated in Fig. 1(a). Using an $x-y$ coordinate system, the initial profile of the grain boundary is given by $y(t=0)=y_{0} \sin (2 \pi x / \lambda)$, at $t=0$, where $\lambda$ is the wavelength and $y_{0}$ the amplitude. Let the houndary energy be $\Gamma$. Then, according to the theory of curvature-driven grain boundary migration, the boundary velocity, $\partial y / \partial t$, is proportional to the curvature $\partial^{2} y / \partial x^{2}$, in a form

$$
\frac{\partial y}{\partial t}=\Gamma M \frac{\partial^{2} y}{\partial x^{2}}
$$

where $M$ is the mobility of the grain boundary. The above equation has a solution

$$
y=y_{0} \sin \frac{2 \pi x}{\lambda} \exp \left(-\frac{4 \pi^{2}}{\lambda^{2}} \Gamma M t\right) .
$$




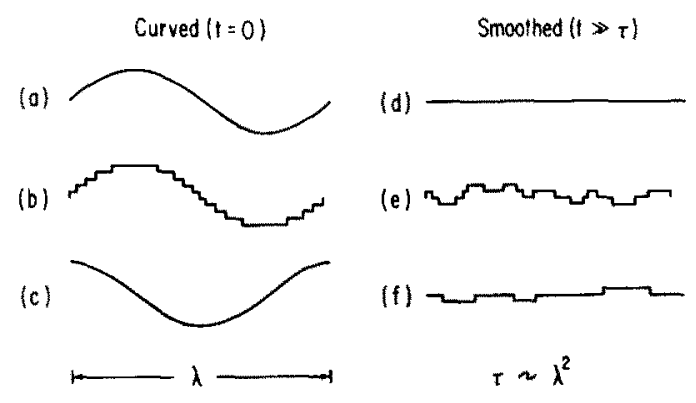

Fig. 1. The initial state $(t=0)$ of a curved boundary is shown in (a) continuum representation and (b) discrete lattice representation. The density of kinks of (b) is plotted in (c). After smoothing $(t \gg 0)$, the grain boundary is again shown in (d) continuum representation and (e) discrete lattice representation. After further recombination of kinks, the smooth boundary (e) becomes (f).

The shape of the boundary at a long time is shown in Fig. 1(d). Thus the decay time $\tau$ for grainboundary smoothing scales with the wavelength in a form of $\tau \sim \lambda^{2}$. We may regard the above kinetics as a variation of the $n=\frac{1}{2}$ type.

Now consider the same boundary in its discrete lattice realization, using a reference square lattice aligned with the $x-y$ coordinate for the upper crystal. Let the lattice parameter be $b$, and let the grain boundary contain straight segments plus kinks of a unit height $b$ at an appropriate spacing, as shown in Fig. 1(b). The density $c$ of such kinks, $1 / b \cdot \partial y / \partial x$, is shown in Fig. 1(c). Here a positive sign refers to a kink with a step upward in the $\hat{y}$ direction, along any straight boundary segment in the $x$ axis. Kinks are allowed to migrate randomly in either direction, in steps of a distance $b$ at a frequency $v_{m}$. Such random walk can smooth out the density profile $c$ as shown in Fig. 1(e). The governing equation for diffusional smoothing is

$$
\frac{\partial c}{\partial t}=\frac{1}{2} v_{\mathrm{m}} b^{2} \frac{\partial^{2} c}{\partial x^{2}}
$$

The equation has the solution

$$
c(t)=c(0) \exp \left(-\frac{2 \pi^{2} v_{\mathrm{m}} b^{2} t}{\lambda^{2}}\right) .
$$

As the density of kinks is smoothed, so is the profile of the grain boundary, which follows

$$
y=y_{0} \sin \frac{2 \pi x}{\lambda} \exp \left(-\frac{2 \pi^{2} v_{\mathrm{m}} b^{2} t}{\lambda^{2}}\right)
$$

by simple conversion between $c$ and $y$. Hence the decay time also scales with $\lambda^{2}$.

The above considerations illustrate that curvalure driven boundary smoothing may actually proceed by the migration of kinks, which is a fluctuation process itself. Indeed, the resultant kinetic law $\lambda \sim \tau^{\frac{1}{2}}$ is identical from either analysis. Since kinked boundaries are more realistic in view of the discrete nature of crystalline lattices, we may view kink motion as a more viable, mechanistic and microscopic description of the boundary motion, regardless of the nature of the driving force.

We can generalize the above picture to polycrystalline grain boundaries. The migration of a kink of positive step height, moving in the $\hat{x}$ direction by $b$, causes the upper crystal to grow by one atom, of an area $b^{2}$, and the lower crystal to shrink by the same amount. If the migration of kinks is random, so must be the growth and shrinkage of grains. In this manner, grains undergo a Brownian motion at a step of $b^{2}$ in either direction. By this mechanism, grain size in a polycrystal can fluctuate. Following Louat [2], the fluctuations of the grain size leads to grain growth.

For specific predictions on the kinetics of grain growth from the Brownian motion of grains, a detailed account of the generation and annihilation of kinks becomes necessary. The simple one-dimensional analysis of Fig. 1 is inadequate for grain aggregates because kinks travel in a ramified network for which the structural description is more involved. Only in the special case of coarsening of orderdisorder domain structure will the one-dimensional analysis suffice. We envision two broad categories, one of a steady state with balanced generation and annihilation of kinks, and the other of a decaying state with the kink density rapidly exhausted due to the lack of nucleation in the presence of kink absorption. These cases will be considered for two- and three-dimensional grain aggregates. We shall use essentially a scaling analysis, in which geometrical constants of the order of unity will be dropped for brevity. For such analysis, the simple geometrical notion of kinks and ledges suffices. After comparison with the computer simulations and experimental data, a more precise physical picture of kinks and ledges in terms of grain boundary dislocations and steps [12] will be presented. These latter considerations will serve to pinpoint the essential difference between grain growth and domain growth [7-11], and to identify the origin of various physical processes affecting kink nuclcation and annihilation in the crystalline solids.

\section{STEADY STATE GRAIN GROWTH UNDER RECOMBINATION CONTROL}

\subsection{2-Dimension}

In 2-dimension, kinks have the following features: (1) they can be nucleated, in a double kink configuration, as shown in Fig. 2(a). The nucleation barrier is $2 \Gamma b$. Once nucleated, the two opposite signed kinks may move apart at no additional expense of energy, or they may recombine with each other. Migration in either direction is equally likely from an energy viewpoint. A unit distance $b$ is travelled in each step of migration. (2) They can pass a triple point where three grains meet, and pass each other, as shown in Fig. 2(b, c). (3) Successive annihilations of opposite kinks reduce the curvature of a 
(a)

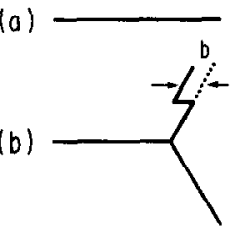

(c)

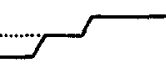

(d)

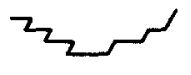

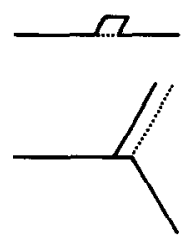
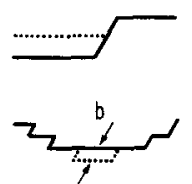
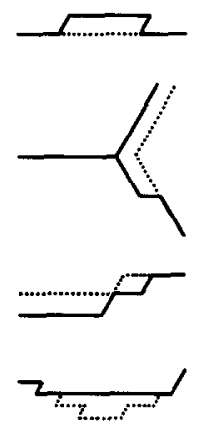

Fig. 2. Kink mechanisms on grain boundaries in a $2 D$ lattice: (a) pair generation, (b) passing a vertice, (c) passing each other, (d) pair annihilations. Each sequence proceeds from left to right. Prior boundaries are traced by dotted lines.

grain boundary, as shown in Fig. 2d. Each step of kink migration adds one atomic area to one of the two bordering grains, while it subtracts the same from the other. If we let $R^{2}$ be the area of the grain, then a fluctuation of $R$ by $\delta=b^{2} / R$ is effected by each step of kink migration. (4) The grain size fluctuation causes a temporal evolution of the grain-size distribution $F(R, t)$. This can be described by a diffusion equation [2]

$$
\frac{\partial F}{\partial t}=\beta \frac{\partial^{2} F}{\partial R^{2}}
$$

where $\beta$ is the effective diffusion coefficient, with $\beta=\frac{1}{2} f_{\mathrm{k}} v_{\mathrm{m}} \delta^{2}$. Here $v_{\mathrm{m}}$ is the migration frequency of the kink and $f_{\mathrm{k}}$ the number of kinks per boundary. This is the same diffusion equation postulated by Louat [2], but he did not identify the fluctuation mechanism.

We envision kinks of either sign to be randomly distributed along grain boundaries in 2-dimension. As shown in Fig. 3, the topology of kink distribution looks different at different kink density. Let the average spacing between kinks be $l$; then the kink density $C_{\mathrm{k}}$ is $l^{-2}$ at low density but $1 / R l$ at high

(a) Low Kink Density

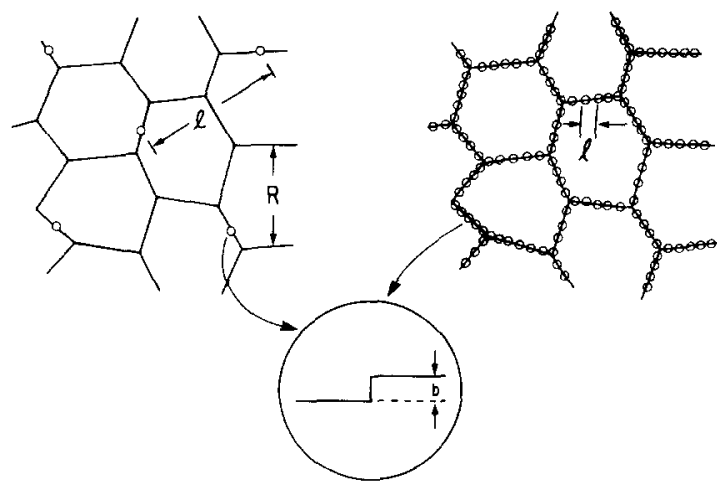

Fig. 3. Distribution of kinks, marked by circles, are shown at (a) low density and (b) high density. density. Now assume kinks are nucleated at a frequency $v_{\mathrm{g}}$ per grain boundary site, and annihilated by mutual recombination and sink absorption. A rate equation is used to describe the evolution of kink density

$$
\frac{\partial C_{\mathrm{k}}}{\partial t}=v_{\mathrm{g}} f_{\mathrm{b}}-C_{\mathrm{k}} / \tau_{\mathrm{r}}-C_{\mathrm{k}} / \tau_{\mathrm{s}} .
$$

In the above, the first term is the generation term, with $f_{\mathrm{b}}$ being the density of grain boundary sites, and $v_{g}$ given by thermal fluctuation over a nucleation barrier. In 2-dimension, $f_{\mathrm{b}}$ is $1 / R b$. The second term is the recombination term in which $\tau_{\mathrm{r}}$ is the half-life of kinks between generation and recombination. Since kinks migrate by random walk in steps of $b$ and the average spacing between them is $l$, a standard random walk analysis gives $\tau_{\mathrm{r}}=(l / b)^{2} / v_{\mathrm{m}}$ as the average time for the two kinks of opposite signs to meet and recombine. The last term is reserved for other sink absorption processes at certain particular lattice sites where kinks can be annihilated. We will explore these possibilities later. For the present, we only specify a certain half life $\tau_{\mathrm{s}}$ for sink absorption. Indeed, we shall let $\tau_{\mathrm{s}}=\infty$ first.

At steady state, $\partial C_{\mathrm{k}} / \partial t=0$. Substitution of $C_{\mathrm{k}}, f_{\mathrm{b}}$, $\tau_{\mathrm{r}}$ in terms of $R, l$, and $v_{\mathrm{m}}$ gives the following solution

$$
\frac{l}{b}=\left\{\begin{array}{cc}
(R / b)^{\frac{1}{4}}\left(v_{\mathrm{m}} / v_{\mathrm{g}}\right)^{\frac{1}{4}} & l \gg R \\
\left(\mathrm{v}_{\mathrm{m}} / v_{\mathrm{g}}\right)^{\frac{1}{3}} & l \ll R
\end{array} .\right.
$$

The above solution suggests a transition at

$$
R^{*}=l^{*}=b\left(v_{\mathrm{m}} / v_{\mathrm{g}}\right)^{\frac{1}{3}}
$$

which is the size scale that the kink spacing becomes comparable to the grain size. The nature of this transition is made clear if we plot the steady state solution in the phase space of $(R, l)$, as shown in Fig. 4. Also indicated there is the line $l=R$ which essentially divides the two regimes. To illustrate the possibility of a transition, the transient evolutions from initial states not lying on the steady state are indicated by arrows in this plot. The above evolutions will be later reflected in the transitions of growth kinetics.

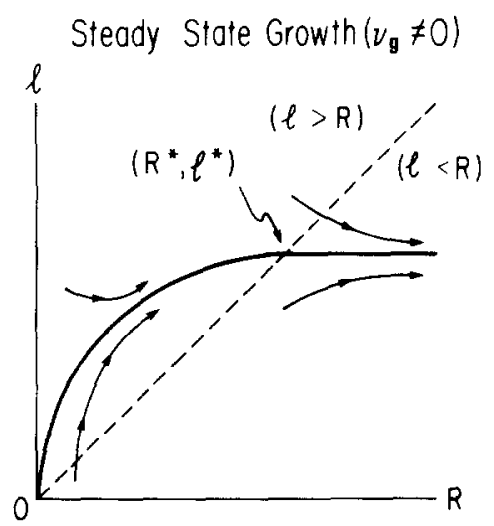

Fig. 4. Phase diagram of grain growth, assuming $v_{\mathrm{g}} \neq 0$. The steady state is the heavy curve. The transient evolutions toward the steady state are shown by arrows. 
The number of kinks per grain is next determined. For $l \ll R$, as in Fig. 3(b), $f_{\mathrm{k}}=R / l$. For $l \gg R$, noting the grain density is $1 / R^{2}$ and the kink density is $1 / l^{2}$, we have $f_{\mathrm{k}}=(R / l)^{2}$.

It follows that the diffusion coefficient in equation (5) is $\beta \sim v_{\mathrm{m}} / R l$ at $l \ll R$ and $\beta \sim v_{\mathrm{m}} / l^{2}$ at $l \gg R$. Explicitly, after substituting equation (7) for $l$, we find

$$
\beta=A v_{\mathrm{m}}\left(v_{\mathrm{g}} / v_{\mathrm{m}}\right)^{p} / R^{q}
$$

where $(p, q)=\left(\frac{1}{3}, 1\right)$ for $l \ll R$ and $(p, q)=\left(\frac{1}{2}, \frac{1}{2}\right)$ for $l \gg R$, and $A$ is a numerical constant of the order of $b^{2+q}$. Thus the diffusion coefficient for grain size fluctuation generally decreases with increasing grain size, particularly so at high kink density. The above dependence is a result of the intrinsic evolutionary nature of kinks which are constantly generated and annihilated along the grain boundary. It does not presume other extrinsic causes such as particle pinning and solute segregation that affect $\beta$ in other ways, although it may be viewed as related to an evolution of the grain boundary structure.

By change of variable, it can be readily shown that a time invariance of the type $t \sim R^{2+q}$ may be admitted into equation (5). If the boundary condition $F(0, t)=F(\infty, t)=0$ is imposed, then the solution to equation (3) necessarily has a characteristic grain size which scales with $t^{n}$, with $n=1 /(2+q)$. The above boundary condition is reasonable because (i) at very small $R$, the curvature and hence the kink density are high; rapid shrinkage of grains is expected, and (ii) very large $R$ is physically unaccessible at any finite time. Incorporating the diffusion coefficient, we find

$$
\bar{R}=B\left(v_{\mathrm{m}} t\right)^{1 /(2+q)}\left(v_{\mathrm{g}} / v_{\mathrm{m}}\right)^{p /(2+q)} b
$$

where $\bar{R}$ is a characteristic grain size, while $B$ is a constant of the order of unity. The time exponent, $1 /(2+q)$, above is $\frac{1}{3}$ for $l \ll R$ and $\frac{2}{5}$ for $l \gg R$.

The grain size distribution is next deduced. Note that in our analysis of the diffusion coefficient, no special account was taken for the size distribution. Hence, self-consistently, we may interpret $R$ in equation (9) as a characteristic grain size which follows equation (10). Upon such substitution, the solution of equation (5) can be obtained easily. The normalized distribution is found to be

$$
F(\tilde{R})=\tilde{R} \exp \left(-\tilde{R}^{2} / 2\right)
$$

where $\tilde{R}=R / \bar{R}$, and $\bar{R}$ is taken to be the grain size at the peak of the distribution. It is worth noting that the normalized distribution is independent of the exponents $q$ and $n$, as long as the time invariance of the type of equation (7) is fully established. Indeed, equation (11) is identical to the distribution that Louat [2] found in the case of constant $\beta\left(n=\frac{1}{2}\right)$.

The above distribution is plotted in Fig. 5, and compared with the distribution from curvature analysis of Hillerts [1] and the empirical log normal distribution of Feltham [13].

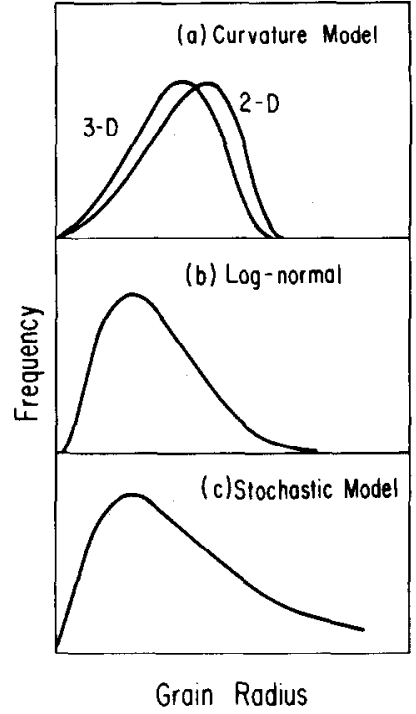

Fig. 5. Grain size distributions according to the (a) curvature model; (b) empirical log-normal fit and (c) stochastic model. Model (a) gives different results in 2 and 3 dimensions.

\subsection{3-Dimension}

The picture of the kink mechanism in 2-dimension can be extended to 3-dimension if we envision ledges of a unit height $b$ lying on the grain boundary. One new feature of ledges is that they will intersect each other to form a network. The mesh size of the network is now denoted as $l$. Ledges can migrate at a unit step $b$, in either direction transverse to the ledge axis. If a ledge maintains its length during migration, there should be no resistance exerted by the nodes of the network. Opposite ledges can mutually annihilate. We assume that double ledges, or ledge loops, of a length comparable to $l$, can be nucleated thermally.

We proceed with the same analysis as in Section 2.1 , starting with equation (6). The distinction between the two cases of $l \ll R$ and $l \gg R$ remains (see Fig. 6). For $l \ll R, C_{\mathrm{k}}=1 / R l^{2}$. For $l \gg R, C_{\mathrm{k}}=1 / l^{3}$. We again denote the nucleation frequency per grain boundary site as $v_{\mathrm{g}}$, and the density of grain boundary sites as $f_{\mathrm{b}}$, now being $1 / R b^{2}$. The half life from a random walk analysis is still $\tau_{\mathrm{r}}=(l / b)^{2} v_{\mathrm{m}}$, where $v_{\mathrm{m}}$ is the migration frequency of the ledge. We let $\tau_{\mathrm{s}}=\infty$ as before. At steady state, the corresponding solution of $l$ is

$$
\frac{l}{b}= \begin{cases}(R / b)^{\frac{1}{5}}\left(v_{\mathrm{m}} / v_{\mathrm{g}}\right)^{\frac{1}{5}} & l \gg R \\ \left(v_{\mathrm{m}} / v_{\mathrm{g}}\right)^{\frac{1}{4}} & l \ll R\end{cases}
$$

and a transition occurs at

$$
R^{*}=l^{*}=b\left(v_{\mathrm{m}} / v_{\mathrm{g}}\right)^{\frac{1}{4}}
$$

The discussion on the nature of the transition made before also applies here. Indeed, the phase diagram of 3-dimension looks essentially identical to Fig. 4, except that the rising portion below $l=l^{*}$ is slightly more concave and further apart from the line of 
(a) Low Ledge Density

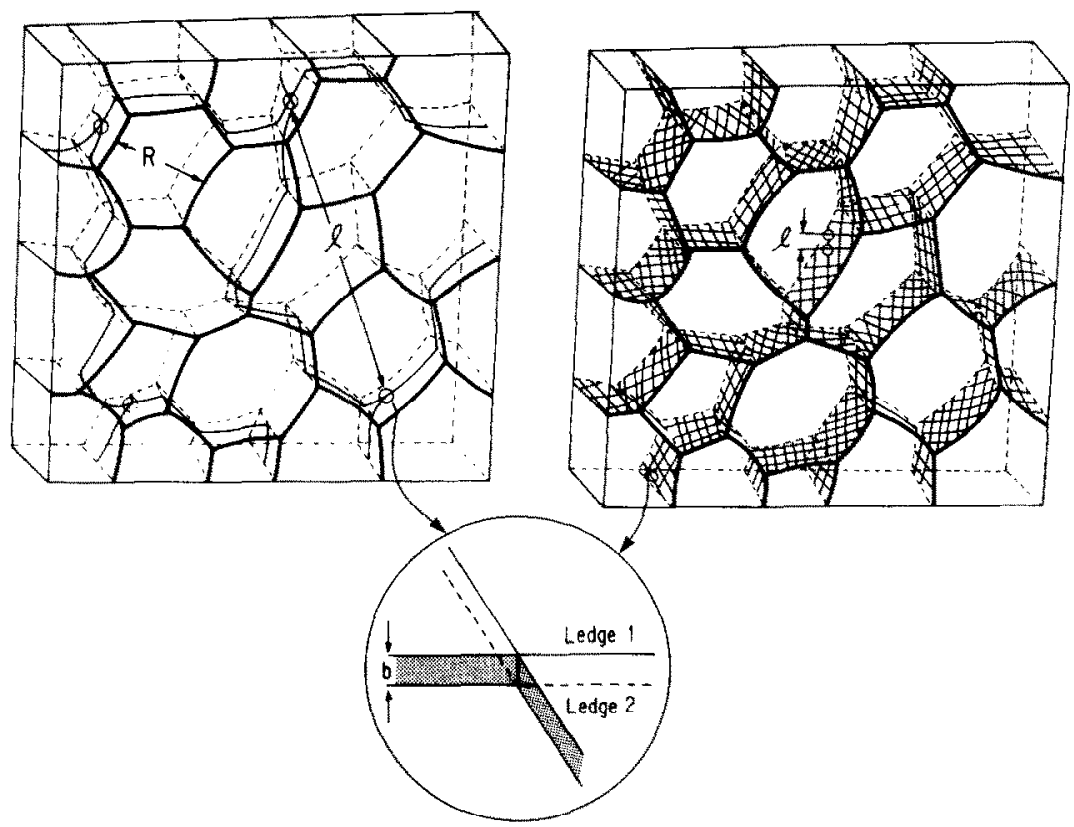

Fig. 6. Distribution of ledges, their intersections marked by circles, are shown at (a) low density and (b) high density.

$l=R$, to reflect the $l \sim R^{\frac{1}{5}}$ dependence of equation (12) as opposed to the $l \sim R^{\frac{1}{4}}$ dependence of equation (7) used previously.

The number of ledges per grain is next determined. For $l \ll R$, referring Fig. $6(\mathrm{~b}), f_{\mathrm{k}}=(R / l)^{2}$. For $l \gg R$, as in Fig. 6(a), $f_{\mathrm{k}}=(R / l)^{2}$. (Coincidentally, $f_{\mathrm{k}}$ here is independent of ledge density.) For $l \gg R$, on each grain boundary traversed by a ledge, the segment of the ledge has a length $R$. This contrasts with the case of $l \ll R$ where the segment of a ledge has a length $l$. Let $R^{3}$ be the volume of each grain, then in a unit step of fluctuation, $\delta=l b^{2} / R^{2}$ is effected by a ledge of $l \ll R$ but $\delta=b^{2} / R$ by a ledge of $l \gg R$.

Using the above results, the diffusion coefficient in equation (5) is found to be $\beta \sim v_{\mathrm{m}} b^{4} / R^{2}$ at $l \ll R$ and $\beta \sim v_{\mathrm{m}} b^{4} / l^{2}$ at $l \gg R$. Explicitly, after substituting equation (12) for $l$, we find the same equation (9) applies, only with the exponents modified as $(p, q)=(0,2)$ for $l \ll R$ and $(p, q)=\left(\frac{2}{5}, \frac{2}{5}\right)$ for $l \gg R$. These results are parallel to those of 2-dimension.

By a similar argument as before, we determine the growth exponent in 3-dimensional grain growth as $\frac{1}{4}$ for $l \ll R$ and $\frac{5}{12}$ for $l \gg R$. However, the normalized grain size distribution given by equation (11), which is independent of the growth exponent, remains the same.

Before closing, a note on equation (5) is warranted. This diffusion equation is strictly applicable only in 1-dimension, hence, for self-consistency, all grain sizes should be interpreted as linear intercepts of grains in using equation (5). Alternatively, appropriate diffusion equations in 2 and 3-dimension may be used, which, in the radial coordinate, differ from equation (5) slightly. The resultant $R(t)$ relations remain the same. The grain size distribution, when $R$ is interpreted as grain radius in 2- and 3-dimension, will differ from equation (11) only in the preexponential factor which must be modified to contain the appropriate power of $R$ for correct normalization. In view of these similarities we have chosen to use the simpler equation (5) in the above analysis.

\section{STEADY STATE GRAIN GROWTH UNDER SINK CONTROL}

In Section 2, we assumed $\tau_{\mathrm{s}}=\infty$, i.e. no sink absorption. We now examine the other limit of sink control.

The relative importance of recombination and absorption is governed by the ratio of $\tau_{r} / \tau_{s}$. Our previous treatment corresponds to the case of $\tau_{s} / \tau_{\mathrm{s}} \rightarrow 0$. When the opposite is true, sink absorption dominates, and retards the growth still further. We can use an alternative interpretation in terms of sink and kink spacings as follows. Since $\tau_{\mathrm{r}}=(l / b)^{2} / v_{\mathrm{m}}$, while $\tau_{\mathrm{s}}$ can be written as $\left(l_{\mathrm{s}} / b\right)^{2} / v_{\mathrm{m}}$ with $l_{\mathrm{s}}$ identified as the sink spacing, the assumption of $\tau_{\mathrm{r}} / \tau_{\mathrm{s}} \rightarrow 0$ is equivalent to $l / l_{\mathrm{s}} \rightarrow 0$, where the spacing of kinks and ledges $l$ is precisely their recombination distance.

For a given sink density, the ratio of $l / l_{\mathrm{s}}$ at the steady state is governed by the kink/ledge generation rate. At high $v_{g}$, which may happen at a high temperature when thermal nucleation of kinks and ledges is easy, equation (7) suggests that $l$ will be small and that $l / l_{\mathrm{s}} \rightarrow 0$. Thus sink absorption is less 
important at high $v_{\mathrm{g}}$ or high temperature. The opposite is true at low $v_{\mathrm{g}}$ or low temperature. Only the latter case needs a new analysis.

Assuming sink absorption to be the dominant annihilation mechanism, i.e. $\tau_{\mathrm{r}}=\infty$, at the steady state we can solve $l$ as before. In 2-dimension, we find

$$
\frac{l}{b}=\left\{\begin{array}{ll}
\left(R b / l_{\mathrm{s}}^{2}\right)^{\frac{1}{2}}\left(v_{\mathrm{m}} / v_{\mathrm{g}}\right)^{\frac{1}{2}} & l \gg R \\
\left(b / l_{\mathrm{s}}\right)^{2}\left(v_{\mathrm{m}} / v_{\mathrm{g}}\right) & l \ll R
\end{array} .\right.
$$

In 3-dimension, we find

$$
\frac{l}{b}=\left\{\begin{array}{ll}
\left(R b / l_{\mathrm{s}}^{2}\right)^{\frac{1}{3}}\left(v_{\mathrm{m}} / \nu_{\mathrm{g}}\right)^{\frac{1}{3}} & l \gg R \\
\left(b / l_{\mathrm{s}}\right)^{2}\left(v_{\mathrm{m}} / v_{\mathrm{g}}\right)^{\frac{1}{2}} & l \ll R
\end{array} .\right.
$$

The phase diagram of Fig. 4 applies for both cases, except that the rising branch is less concave and closer to the line of $l=R$, to reflect the $l \sim R^{\frac{1}{2}}$ and $l \sim R^{\frac{1}{3}}$ dependence here. The transition at $l^{*}=R^{*}$ is obviously given by the values of $l$ at $l \ll R$. The similarity of these results and those of recombination control is obvious, since both $\tau_{\mathrm{r}}$ and $\tau_{\mathrm{s}}$ are proportional to $v_{\mathrm{m}}^{-1}$. Lastly, re-examination of the growth law finds that the growth exponents remain the same as those for recombination control at $l \ll R$, but become $\frac{1}{3}$ in 2-dimension and $\frac{3}{8}$ in 3-dimension at $l \gg R$.

\section{RETARDED GRAIN GROWTH}

Until now, we have assumed that kinks and ledges can be generated by thermal fluctuation. This may not be possible, especially in 3-dimension, due to the relatively large nucleation barrier. Nevertheless, an initial density of kinks and ledges may be present which then evolves according to equation (6) with $v_{\mathrm{g}}=0$. We still assume $\tau_{\mathrm{s}}=\infty$ here for the moment. This situation with continuously depleting kinks and ledges is expected to lead to very slow grain growth.

Noting that $\tau_{\mathrm{r}}=(l / b)^{2} / v_{\mathrm{m}}$ in both 2- and 3-dimensions, we reduce, at $v_{\mathrm{g}}=0$ and $\tau_{\mathrm{s}}=\infty$, equation (6) to

$$
\frac{\partial C_{\mathrm{k}}}{\partial t}=-\frac{v_{\mathrm{m}} b^{2}}{l^{2}} C_{\mathrm{k}}
$$

The above equation gives a scaling $l / b \sim\left(v_{\mathrm{m}} t\right)^{\frac{1}{2}}$ in all cases. Note further that in 2-dimension, $\beta \sim v_{\mathrm{m}} b^{4} / R l$ at $l \ll R$ and $\beta \sim v_{\mathrm{m}} b^{4} / l^{2}$ at $l \gg R$, while in 3-dimension, $\beta \sim v_{\mathrm{m}} b^{4} / R^{2}$ at $l \ll R$ and $\beta \sim v_{\mathrm{m}} b^{4} / l^{2}$ at $l \gg R$. After substituting $\left(v_{\mathrm{m}} t\right)^{\frac{1}{2}}$ for $l$, we find that the following diffusion equation holds at $l \gg R$ in both two and three dimensions

$$
\frac{\partial F}{\partial t}=A\left(b^{2} / t\right) \frac{\partial^{2} F}{\partial R^{2}}
$$

where $A$ is again used as a constant, of the order of unity in this case.

It can be easily shown that the time invariance of the solution of the above equation is logarithmic, $R^{2} \sim \ln t$. Very slow grain growth is thus expected in this limit. The kink density and ledge density vary as $(l / b)^{2} \sim \exp (R / b)^{2}$.

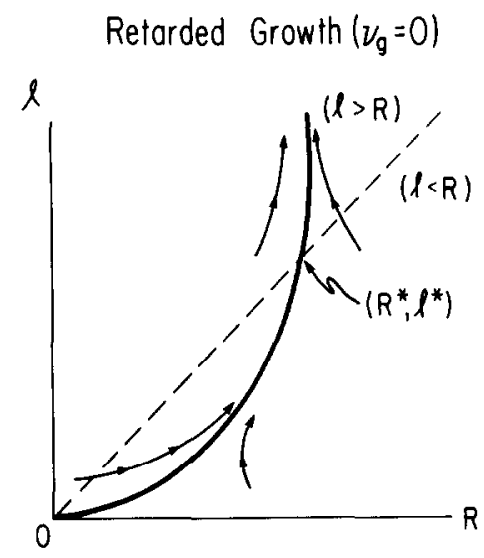

Fig. 7. Phase diagram of grain growth, assuming $v_{\mathrm{g}}=0$. The steady state is the heavy curve. The transient evolutions toward the steady state are shown by arrows.

At the other limit, $l \ll R$, we find the time invariance in 2-dimension to be $R \sim b\left(v_{\mathrm{m}} t\right)^{\frac{1}{6}}$, and in 3-dimension $R \sim b\left(v_{\mathrm{m}} t\right)^{\frac{1}{4}}$, following a similar reasoning. Hence $(l / b) \sim(R / b)^{3}$ in 2-dimension and $(l / b) \sim(R / b)^{2}$ in 3-dimension in this limit.

We plot these solutions in the phase space. In Fig. 7 , the branch of the exponential rise is at the $l \gg R$ limit, and the branch near the origin is at the $l \ll R$ limit. Note that the shape of the curve of retarded grain growth of Fig. 7 is very different from that of steady state grain growth of Fig. 4. Topologically, the two are mirror images of each other with respect to $l=R$.

If the initial density of kinks and ledges is high enough so that sink absorption is at first unimportant, the above analysis applies too. However, as $l \sim\left(v_{\mathrm{m}} t\right)^{\frac{1}{2}}$, eventually $l>l_{\mathrm{s}}$ and sink absorption dominates. From then on, the density of kinks and ledges will be depleted exponentially, and a complete arrest of grain growth must occur. This situation can be depicted using the same phase diagram of Fig. 7 , except that the rapidly rising branch there now approaches a terminal $R$ asymptotically. However, finite exponents can be obtained for sink control and $l \ll R$ (see Table 1 below).

In all of the above cases, the normalized grain size distribution of equation (11) is not altered. This can be shown for equation (15), for example, by a simple change of variable from $t$ to $\ln t$, upon which equation (15) reduces to the standard diffusion equation.

Table 1. Exponents of grain growth $(n, m)^{a}$

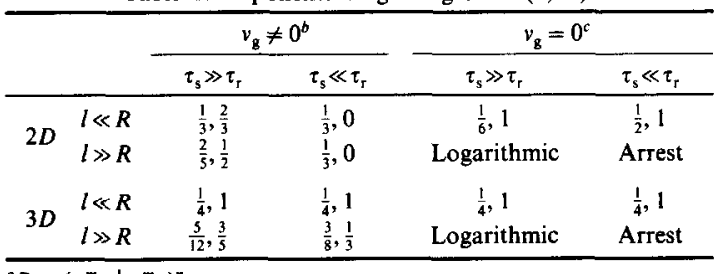

${ }^{a} R \sim\left(v_{\mathrm{m}}^{m} v_{\mathrm{g}}^{I-m_{t}}\right)^{n}$.

${ }^{b}$ Phase diagram is concave downward in $(R, l)$ coordinate. See Fig. 4.

${ }^{c}$ Phase diagram is concave upward in $(R, l)$ coordinate. See Fig. 7. 
The solution of grain growth kinetics is now complete. We write the growth law as $R \sim\left(v_{\mathrm{m}}^{m} v_{\mathrm{g}}^{1-m} t\right)^{n}$, where $n$ is the growth exponents and $m$ may be used to infer the activation energy through $v_{\mathrm{m}}$ and $v_{\mathrm{g}}$. These exponents are tabulated in Table 1. In the special case of logarithmic growth or total arrest, $n=0$ and no temperature dependence is manifested. In all cases, only one universal grain size distribution applies.

In examining Table 1 , note that there can be a cross over from the case of $v_{\mathrm{g}}=0$ to $v_{\mathrm{g}} \neq 0$. For example, if the rates of recombination and sink annihilation are initially large in comparison with that of generation, it may be assumed that $v_{\mathrm{g}}=0$ at first. As the densities of kinks and ledges decrease, the rates of recombination and sink annihilation also decrease rapidly. Eventually, even a small amount of generation becomes significant, hence $v_{\mathrm{g}} \neq 0$ at a later stage. More detailed considerations of such cases will be left for future investigations.

\section{COMPARISON WITH SIMULATION AND EXPERIMENTAL RESULTS}

\subsection{Comparison with simulation results}

Recently, a series of Monte Carlo simulations of domain growth in highly degenerate Potts systems has been reported [7-11]. When a triangular lattice is used, such that the grain vertices do not behave as sinks for kinks, these models are offered by their investigators as representative of steady state grain growth in 2-dimension. Specifically, they found that the growth exponents are 0.33 and $0.41 \pm 0.02$, at the short time and the long time limit. These results are in excellent agreement with our prediction in Table 1 $\left(\frac{1}{3}\right.$ and $\frac{2}{5}$ ). In 2-dimension, the investigators also found [10] that their grain size distribution was nearly identical to that predicted by Louat [2], namely equation (11).

In 3-dimension, the simulations found only one exponent, $0.37 \pm 0.02$ [11]. While the investigators also found Louat's prediction of grain size distribution [equation (11)] to be close to what they observed, a prominent secondary peak was initially reported at very small grain sizes [11]. The latter has since been found to be an artifact due to the way they originally performed their cluster analysis [14]. Furthermore, it was found that vertices in the 3-dimensional lattice employed in their study absorb kinks [14]. We predicted that sink absorption will eventually dominate unless $v_{\mathrm{g}}$ is very high. The latter possibility was unlikely in these simulations, which were carried out at $T \simeq 0$. Hence, we predict an exponent $\frac{3}{8}$ for the present case, again in excellent agreement with their result.

The nature of the transition in 2-dimension requires clarification. These simulations were conducted using an initial state which was quenched from the melt state to $T \simeq 0$. The very large undercooling resulted in a very small initial grain size, $R \simeq b$. If we further assume $R>l$ at the initial state, then the initial exponent in 2-dimension should indeed be $\frac{1}{3}$. According to the phase diagrams of Fig. 4, however, this initial state must evolve toward the steady state. In doing so, it crosses the boundary at $l \simeq R$ and, from then on, assumes the other exponent $\frac{2}{5}$. In these simulations, this transition occurred at $R \simeq 6 b$. Subsequently, it should follow the steady state on the rising branch of Fig. 4. Because $v_{\mathrm{m}} \gg v_{\mathrm{g}}$ in these simulations, the other transition at $l^{*}=R^{*}$ probably was never reached when simulations were terminated at $R \simeq 30 b$ [7-10]. Hence the exponent remains as $\frac{2}{5}$. It appears plausible that the same situation could have been encountered in 3-dimension simulations as well, although the complexity of initial transients involving recombination and sink absorption, or perhaps an insufficient numerical resolution, seemed to have obscured a similar transition in 3-dimension.

Other than the triangular lattice, the same investigators also examined a square lattice in 2-dimension in which they found an arrest of grain growth at low temperatures [7-9]. At higher temperatures, the results obtained from a square lattice have no arrest and the exponents are identical to that of the triangular lattice. The anomaly at low temperatures was attributed to the absorption of kinks at the grain vertices in a square lattice. This behavior can be easily understood using our analysis in Sections 3 and 4 . As shown in our analysis, sink absorption will be controlling when the kink density is low and will be negligible when the opposite is true. At low temperatures, when kink generation is sluggish, we expect sink absorption to lower the density of kinks significantly further. Near absolute zero degrees temperature, $v_{\mathrm{g}}=0$; hence, sink absorption results in a complete arrest of the growth. This is indeed observed in simulations. At high temperature, on the other hand, kink generation is rapid enough to maintain a high density of kinks. Under such circumstances, sink absorption is secondary and may be ignored. The growth exponents are thus independent of lattice types at higher temperatures, as the simulations indicated. The topology of the cubic lattice used in their 3-dimensional simulations, though, appears to allow ledge absorption at vertices at least at modestly high temperatures [14].

As already mentioned, our model at $v_{\mathrm{g}} \neq 0$ predicts a long-time exponent of $n=1 /(d+1)$, where $d$ is the dimensionality. While this prediction was not realized in simulations, presumably due to size and time limitations, it is in agreement with the theoretical expectation based on cluster thermodynamics. Using a droplet model, Furukawa [15] pointed out that the entropic term of the droplet free energy due to surface roughening, which is proportional to $k T \ln R$, should give rise to a long-term droplet growth exponent of $n=1 /(d+1)$. In our model, in the same thermodynamic limit, we expect that $l$ approaches a constant much smaller than the grain size, and that kinks of 
such spacing cause roughening of grain boundaries. Thus physically the two pictures correspond to each other and should give the same prediction.

We acknowledge here that much of the kink/ledge mechanisms described in this work were recognized by Sahni et al. [7-11]. While they realized the central role of such mechanisms in domain growth in the simulations, they did not crystallize the idea to present a quantitative treatment. The present analysis, by ignoring some of the topological details of the real polycrystals, is able to quantify the kink/ledge mechanism and to relate it to growth kinetics. It nevertheless shares the same physical basis as the simulations, and both may be characterized as an atomistic description of domain and grain growth.

From the above comparison, we may conclude that the present theory accurately captures the essence of the highly degenerate Potts model. [The other limit, i.e. the Ising model in which no triple point exists, is adequately described by equation (3-4).] Indeed, these two probably are physically equivalent approaches. However, the Potts model, strictly speaking, is applicable only to domain growth and not to grain growth, while our model has no such restrictions, provided appropriate physical mechanisms and numerical values are assigned to the generation, recombination, and absorption processes. We shall return to this subject in Section 6.

\subsection{Comparison with experimental results}

Several grain-growth studies on zone refined metals have been reported in the literature. However, no common grain growth exponent was found in these studies. For lead and tin, the growth exponent was found to be between 0.40 and 0.43 [16-18], although the exponent $\frac{1}{2}$ was also reported for tin [18] in one study. In the latter study, it was noticed [18] that the scatter of experimental points was relatively large in zone refined metals, but was reduced when impurity was added. The cause of the scatter was not identified. It was often reported, for example, for lead [16] that an ultimate grain size is of the order of sample thickness. For both lead and tin, a transition to a smaller exponent was reported [17]. For aluminum, a considerably lower exponent, around 0.25 [19], was found. At slightly lower purity and at longer annealing time, the exponent was even smaller [19]. Lastly, for zone refined iron, the exponent was found to increase from approximately 0.25 to 0.5 as the temperature increased from 550 to $850^{\circ} \mathrm{C}$ [20]. Typically, these experiments were conducted using polycrystals with an initial grain size of the order of $0.1 \mathrm{~mm}$. An increase of one order of magnitude or less in the grain size was studied.

The relative coarse initial grain sizes in these studies makes it apparent that, unlike Monte-Carlo simulations [7-11], these growth experiments did not begin with a quenched state. The first transient caused by the crossing of the border of $l=R$ from below in Fig. 4 was therefore not experienced. In general, the relatively narrow range of the grain size studied experimentally makes it difficult to capture the transition between the two regimes of $l \ll R$ and $l \gg R$. Depending on the initial grain size, the values of $l^{*}=R^{*}$, and the relative importance of ledge nucleation and sink absorption, grain growth may evolve along the steady state with an exponent of 0 , $\frac{1}{4}, \frac{3}{8}$ or $\frac{5}{12}$. In particular, the sometimes observed retardation in the long time can be caused by the onset of sink control, especially as the grain size approaches the sample thickness (see next section). It appears that the upper value of these exponents, $\frac{5}{12}$, was seen in lead, tin and iron, and the lower value of $\frac{1}{4}$ was seen in aluminum.

Although the comparison of our model and the observed grain growth exponents is not definitive, the grain size distribution, which is well-fitted by equation (11), was indeed observed in Ref. [20] for iron. Many other investigators of grain growth have followed the suggestion of Feltham [13] to fit their data on grain size distribution to a log normal form. The latter is graphically quite similar to equation (11), both with a tail at the larger sizes (Fig. 5). By implication, it may be inferred that these data, which are not necessarily for high purity metals, are consistent with equation (11). In contrast, Hiller's curvature driven growth model [1], like that of the Lifshitz-Slyozov-Wagner theory of precipitate coarsening $[21,22]$, predicted a size distribution with a short tail at the larger sizes (Fig. 5). So the comparison on grain size distributions favors the stochastic model presented here. Specifically, the universality of the size distribution, irrespective of the growth exponent, is in agreement with our model.

Associated with the multiplicity of the growth exponent, our model predicts a range of activation energy to be governed by $\left(v_{\mathrm{g}}^{1-m} v_{\mathrm{m}}^{m}\right)^{n}$ from Table 1 . It will become clear in the next section that the activation energy of $v_{\mathrm{m}}$ should be that of grain boundary diffusion, but that of $v_{\mathrm{g}}$ can vary widely. In the literature, there is no definitive experimental evidence that the activation energy of grain growth is precisely that of grain boundary diffusion, although most results suggest that the two activation energies are of similar magnitude. The above experimental observations are not inconsistent with the prediction.

\section{DISCUSSIONS}

While the present model is a drastic departure from the conventional treatment of normal grain growth based on the curvature driven mechanism, it stands as a structural rationalization and generalization of the stochastic model of Louat's [2]. The essence of the model is that kinks and ledges are the microstructural units which are responsible for grain boundary migration. The realization of the picture is straightforward in the Potts model for domain growth, in which atoms on both sides of a domain boundary assume the same lattice structure, their only 


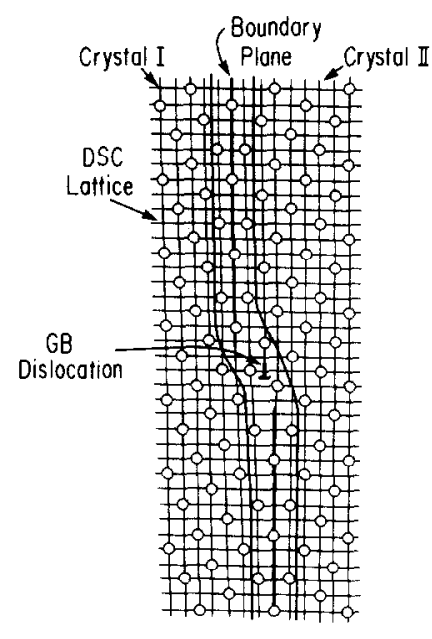

(a)

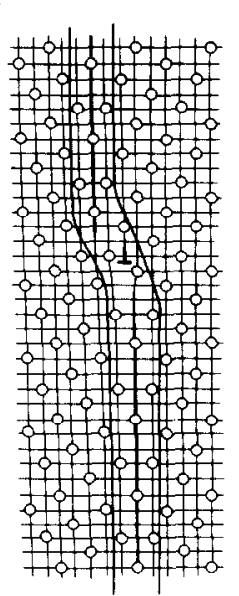

(b)
Fig. 8. Grain boundary dislocation at a step in symmetric tilt boundary in simple cubic structure (tilt angle $=36.9^{\circ}$ ). (a) Initial structure (b) after climb by annihilation of one vacancy.

difference being their spins. In grain growth, however, the lattices on the two sides of a grain boundary are rotated and translated with respect to each other. In this case, kinks and ledges should be identified as those grain boundary dislocations associated with the stepped structure, as shown in Fig. 8, for example. Such structures have been extensively studied in recent years [23, 24], and a catalog of step heights and Burgers vectors is known for cubic metals and coincidence boundaries $[12,25]$. Since any random boundary may be regarded as an ordered array of various types of grain boundary dislocations [26], the existence of stepped structures should be a rather general feature. While this picture has so far been pursued mostly for macroscopically flat boundaries, its incorporation in curved boundaries is conceptually straightforward and consistent with the simplified representations used in the present paper. The spacing of the stepped structure should then be a function of the curvature as well as the misorientation of the boundary, and it is those extraneous grain boundary dislocations not belonging to the macroscopically flat boundaries that correspond to kinks and ledges in our model. Of course, in a more detailed treatment, the multiplicity of different kinks and ledges possessing different step heights and different widths, and the manifestation of various conservation laws governing grain boundary dislocations, should also be considered. However, these details are not expected to alter the basic formulation of our rate theory based on equation (4) and our fluctuation theory based on equation (3).

In real grain growth, migration of ledges, or equivalently, migration of grain boundary dislocations and steps, always requires diffusion of defects [12]. The current understanding of grain boundary diffusion supports the idea that vacancy is the dominant defect. Assuming detailed balance, it can be readily argued that the rate of vacancy absorption is statistically equal to that of vacancy emission at grain boundary dislocations. Hence the climb of grain boundary dislocations should not be biased, a priori, in either direction. Thus, the assumption that $v_{\mathrm{m}}$ can be used to describe random migration of ledges is justified. Indeed, $v_{\mathrm{m}}$ should scale with $D_{\mathrm{gb}} / b^{2}$ where $D_{\mathrm{gb}}$ is the self-diffusivity along grain boundaries. The above conclusion may be altered if the climb of grain boundary dislocations is biased due to a gradient of defect concentrations or alloy compositions. (Diffusional creep is an example of the former [27]; diffusion induced grain boundary migration is an example of the latter [28]. A combination of both can be envisioned [29].) In normal grain growth of unstressed metals and possibly of uniform alloys, such bias does not exist.

The plausibility of thermal nucleation of ledges can now be discussed. Nucleation of ledges by defect condensation has been observed in irradiated, supersaturated metals [30]. However, the special condition under which the above observation was made underlines the general difficulty of thermal nucleation of grain boundary dislocations in internal grain boundaries in equilibrated crystals. (The extreme difficulty of thermal nucleation of lattice dislocations is wellknown. Even though grain boundary dislocations have a smaller Burgers vector in all cases, their thermal nucleation is still quite difficult.) Thus, quite likely, most of the ledges which participate in grain growth are "quenched-in" ones as a result of prior thermal mechanical treatment.

At higher temperatures, however, increasing disorder of grain boundary in the form of increasing structural unit multiplicity and increasing vibrational amplitudes and anharmonicity may be expected. These effects may cause a spreading of the cores of grain boundary dislocations that lowers their nucleation barriers. Thus the analysis of steady state grain growth in Sections 2 and 3 becomes more applicable. Indeed, if the theoretical suggestion of more extended disordering (melting) transition of grain boundary [31-35] is to be verified at very high temperatures, the notion of kinks and ledges might be disposed of entirely. Grain boundaries may be curved on an atomic scale, with a largely disordered liquid-like structure. Only in this extreme can grain growth be rationalized by curvature driven growth, with grain boundary migrating as a whole by atomic transport across the entire liquid-like layer. The growth exponent of $\frac{1}{2}$ becomes applicable here. (A soap foam coarsens in precisely this manner, for which the classical exponent of $\frac{1}{2}$ was experimentally confirmed [36].) On the other hand, the rarity of this exponent's being observed in grain growth, in high purity crystalline materials and at high temperatures, could implicate the rarity of grain boundary melting in real materials, e.g. nominally pure aluminum [37].

In view of the difficulty of thermal nucleation, it has sometimes been assumed [38] that a spiral mech- 
anism $[39,40]$ analogous to the screw dislocation mechanism [41] in condensation and solidification could operate on the grain boundary, providing a fresh supply of kinks and ledges. The source of the spirals is attributed to extrinsic lattice dislocations impinging a grain boundary with a normal Burgers vector. Such dislocations are obviously available from the thermal mechanical treatment prior to grain growth. However, spirals are known to maintain the maximal, critical curvature near the origin. In the case of grain growth, provided the driving force is that of grain boundary curvature, it can be readily shown from the standard relation between the critical spiral radius and the driving force [39] that the minimal radius of the spiral is of the order of grain size itself. Moreover, spiral sources will be rendered inoperative if their spacing is shorter than the critical radius [42]. In the latter case, they behave very much as an inoperative Frank-Read source [43], due to the lack of a sufficient driving force. Since dislocation density in a typical metal is at lest $10^{12} / \mathrm{m}^{2}$ during grain growth, most spiral sources are spaced at distances smaller than the typical grain size, such that the spiral mechanism itself is not likely to operate efficiently to regenerate grain boundary dislocations. On the other hand, as grain boundaries fluctuate and eventually sweep by a substantial volume, they absorb new lattice dislocations which, by dissociation [44], provide a fresh supply of grain boundary dislocations. The kinetics of the above process are difficult to estimate, a priori, because of the evolutionary nature of the lattice dislocations which undergo recovery themselves. (In a typical grain growth experiment, grain size increases by more than tenfold, hence only one one-thousandth of the original grains survive. It is obvious that the grain interior has been swept by grain boundaries many times, making it unlikely to maintain a constant dislocation density.) Consequently, an accurate estimate of $v_{\mathrm{g}}$ will not be attempted here.

Grain growth under dynamic conditions can be grcatly enhanced by virtuc of a more plentiful supply of new ledges. High intensity displacement damage by irradiation should produce such results. Another notable example, in our opinion, is that of enhanced grain growth in superplastically deformed fine-grain polycrystals. It is interesting to note that a theory similar to that of Louat's [2] has recently been proposed for the latter phenomenon [45].

The other aspect of kink/ledge kinetics is sink annihilation. Under normal circumstances, internal surfaces, such as the surfaces of voids or the incoherent interfaces of precipitates and dispersoids, are the most likely sinks of grain boundary dislocations. (Here also lies another essential difference between domain growth and grain growth, in that grain boundary dislocations have an elastic stress field and are attracted to free surfaces and incoherent interfaces. Such long-range attraction does not exist for kinks and ledges on domain boundaries, where only the nearest-neighbor bondings between rigid lattice sites are considered.) This provides an alternative interpretation of grain boundary pinning and the eventual arrest of grain growth by particles and voids [3-6]. External surfaces can also become the dominant sinks themselves. This situation will be encountered in thin polycrystals, when grains grow to the size of the sample. It is well known that when columnar grains spanning the entire thickness of thin plates or films have formed, the normal grain growth is arrested. Rather than adopting surface grooving (as in the curvature-driven grain-growth theory) as the cause [46], we offer sink absorption at external surfaces as an alternative physical mechanism.

Until now, we have oversimplified one aspect of our analytical treatment of grain growth in the discussion. We now address this point before closing. We argued that there is an essential topological transition concerning the evolution of the kink spacing and the grain size, depending on their relative lengths. More rigorously, this transition can be defined as when a kink on a grain edge has equal probability during a random walk to encounter another kink (a) on the same grain edge and (b) on a different edge. Using a 2-D polycrystal of periodic hexagons as a reference, we can verify that the transition corresponds to the case when each edge has two kinks. At a uniform kink spacing, the above gives $R=2 \sqrt{3} l$ at the transition if the grain size is taken as the distance between two nearest parallel edges. A relative grain size much larger than the above corresponds to the case of $R \gg l$ in the text, etc. A similar argument can be made for 3-D polycrystals which, presumably, will give analogous results.

Acknowledgement-This research was supported by U.S. National Science Foundation Grant No. DMR-8407868.

\section{REFERENCES}

1. M. Hillert, Acta metall. 13, 227 (1965).

2. N. P. Louat, Acta metall. 22, 721 (1974).

3. K. Lücke and K. Detert, Acta metall. 5, 628 (1957).

4. J. W. Cahn, Acta metall. 10, 789 (1962).

5. C. Zener, private communication to C. S. Smith, Trans. metall. Soc. A.I.M.E., 175, 15 (1949).

6. T. Gladman, Proc. R. Soc. A294, 298 (1966).

7. P. S. Sahni, G. S. Grest, M. P. Anderson and D. J. Srolovitz, Phys. Rev. Lett. 50, 263 (1983).

8. P. S. Sahni, D. J. Srolovitz, G. S. Grest, M. P. Anderson and S. A. Safran, Phys. Rev. B28, 2705 (1983).

9. M. P. Anderson, D. J. Srolovitz, G. S. Grest and P. S. Sahni, Acta metall. 32, 781 (1984).

10. D. J. Srolovitz, M. P. Anderson, P. S. Sahni and G. S. Grest, Acta metall. 32, 793 (1984).

11. M. P. Anderson, G. S. Grest and D. I. Srolovitz, Scripta metall. 19, 225 (1985).

12. R. W. Balluffi, in Grain Boundary Structures and Kinetics (edited by R. W. Balluffi), p. 297. Am. Soc. Metals, Metals Park, Ohio (1980).

13. P. Feltham, Acta metall. 5, 97 (1957).

14. M. P. Anderson, private communication (1985).

15. H. Furukawa, Phys. Rev. A29, 2160 (1984).

16. G. F. Bolling and W. G. Winegard, Acta metall. 6, 283 (1958). 
17. J. P. Drolet and A. Galibois, Acta metall. 16, 1387 (1968).

18. E. L. Holmes and W. C. Winegard, Acta metall. 7, 411 (1959).

19. P. Gordon and T. A. El-Bassyouni, Trans. metall. Soc. A.I.M.E. 233, 391 (1965).

20. H. Hu, Can. Metall. Quant. 13, 275 (1974).

21. I. M. Lifshitz and V. V. Slyozov, Zh. Ekxp. Teor. Fiz 35, 479 (1958).

22. C. Wagner, Z. Electrochem. 65, 581 (1961).

23. For a review, see Structure and Property of Internal Interfaces, J. Physique 46, C4 (1985).

24. A. H. King and D. A. Smith, Acta crystallogr. A36, 335 (1980).

25. A. H. King, Acta metall. 30, 419 (1982)

26. A. P. Sutton and V. Vitek, Phil. Trans. R. Soc. Lond. A309, 1, 37, 55 (1983).

27. B. Button, Diffusional Creep of Polycrystalline Materials Trans. Tech. Publ., Bay Village, Ohio (1977).

28. R. W. Balluffi and J. W. Cahn, Acta metall. 29, 493 (1981).

29. I-W. Chen, Acta metall. 30, 1317 (1982).

30. A. H. King and D. A. Smith, Phil. Mag. 42A, 495 (1980).

31. R. Kikuchi and J. W. Cahn, Phys. Rev. B21, 1983 (1980).
32. G. Ciccotti, M. Guillope and V. Pontikis, Phys. Rev. B27, 5576 (1983).

33. F. Carrion, G. Kalonji and S. Yip, Scripta metall. 17, 915 (1983).

34. G. Kalonji, P. Deynier, R. Najafabadi and S. Yip, Surf. Sci. 144, 77 (1984).

35. P. S. Ho, T. Kwok, T. Nguyen, C. Nitta and S. Yip, Scripta metall. 19, 933 (1985)

36. J. C. Fisher, private communication to R. L. Fullman, in Metal Interfaces, p. 179 Am. Soc. Metals, Cleveland, Ohio (1952).

37. S-W. Chan, J. S. Liu and R. W. Balluffi, Scripta metall. 19, 1251 (1985)

38. D. A. Smith and C. M. F. Rae, Metal Sci. 13, 101 (1979).

39. J. W. Christian, Theory of Transformations in Metals and Alloys. Pergamon Press, Oxford (1965).

40. B. Burton, Mater. Sci. Engng 10, 9 (1972).

41. F. C. Frank, Adv. Phys. 1, 91 (1953).

42. W. K. Burton, N. Cabrera and F. C. Frank, Phil. Trans. A243, 299 (1950)

43. J. P. Hirth and J. Lothe, Theory of Dislocations. Wiley, New York (1982).

44. R. C. Pond and D. A. Smith, Phil. Mag. 36, 353 (1977).

45. $H$. Frost and $R$. Raj. To be published.

46. W. W. Mullins, J. appl. Phys. 27, 900 (1956). 